\title{
Hardness and excitation energy
}

\author{
Á NAGY \\ Department of Theoretical Physics, University of Debrecen, and Atomic and Molecular Physics Research \\ Group of the Hungarian Academy of Sciences, H-4010 Debrecen, Hungary \\ e-mail: anagy@dtp.atomki.hu
}

\begin{abstract}
The concept of the ensemble Kohn-Sham hardness is introduced. It is shown that the first excitation energy can be given by the Kohn-Sham hardness (i.e. the energy difference of the ground-state lowest unoccupied and highest occupied levels) plus an extra term coming from the partial derivative of the ensemble exchange-correlation energy with respect to the weighting factor $w$ in the limit $w \rightarrow 0$. It is proposed that the first excitation energy can be used as a reactivity index instead of the hardness.
\end{abstract}

Keywords. Kohn-Sham hardness; excitation energy; reactivity index.

\section{Introduction}

The concepts of chemical reactivity theory have been given a rigorous foundation in the density functional theory ${ }^{1-4}$ from Parr and collaborators. ${ }^{2}$

In the density functional theory the total energy $E[n]$ is a unique functional of the density $n$. Parr et al consider the total energy $E$ a function of the number of electrons $N$. The chemical potential is defined as the negative of the electronegativity

$$
\mu=(\partial E / \partial N)_{\mathrm{v}}=-\chi .
$$

It was long ago showed by Mulliken ${ }^{5}$ that the electronegativity of a species can sensibly be defined as the average of its ionization potential $I$ and electron affinity $A$

$$
\chi_{M}=(I+A) / 2 .
$$

This is just the finite difference approximation to (1). From the variational principle of the density functional theory follows Sanderson's principle: ${ }^{6}$ the electronegativity equalizes when two species unite to form a new species leading to a single electronegativity or chemical potential (the same way as in ordinary thermodynamics).

The hardness $\eta$ of an electronic system is defined ${ }^{7}$ as

$$
\eta=\frac{1}{2}\left(\frac{\partial \mu}{\partial N}\right)_{V}=\frac{1}{2}\left(\frac{\partial^{2} E}{\partial N^{2}}\right)_{V}
$$

The global softness ${ }^{8}$ is the inverse of the global hardness

$$
S=\frac{1}{2 \eta}=\left(\frac{\partial N}{\partial \mu}\right)
$$

It can be easily shown ${ }^{2}$ that the global hardness can be approximated by

$$
\eta \approx(I-A) / 2 .
$$

It can be further approximated by the Kohn-Sham hardness, that is the energy difference of the lowest unoccupied and the highest occupied molecular orbitals:

$$
\eta \simeq \eta_{\mathrm{KS}}=\frac{1}{2}\left(\varepsilon_{\mathrm{LUMO}}-\varepsilon_{\text {Номо }}\right) .
$$

Electronegativity, hardness and softness have proved to be very useful quantities in the chemical reactivity theory. Nevertheless, the definitions above cannot be applied with complete rigour. For example, there are several different definitions for the chemical potential within the present density functional framework. ${ }^{9}$ In their recent paper, Zahariev and Wang ${ }^{9}$ have proved that at an integer electron number, for any external potential converging to the same constant at infinity in all direction, the value of the chemical potential is the negative of the first ionization energy

$$
\mu=-I \text {. }
$$

They also rigorously derived within the framework of zero-temperature Fock-space density functional theory that the chemical potential is equal to the Lagrangian multiplier used to constrain the normalization of the density in the traditional density functional theory.

As it was emphasized by Cohen in his important contribution to 'Strengthening the Foundation of 
Chemical Reactivity', ${ }^{10}$ the fact that finite systems have discrete energy spectra has important consequences for the reactivity theory. The values of softness and hardness are either zero or infinity.

In this paper, based on the ensemble theory of excited states, a relationship between excitation energy and hardness is derived. It is proposed that the first excitation energy can be used as a reactivity index instead of the hardness. Finally, the concept of the ensemble Kohn-Sham hardness is introduced.

\section{Ensembles of excited states}

First the theory of excited states for ensembles by Gross et $a l^{12}$ is reviewed. The first rigorous generalization of time-independent density functional theory for excited states was given by Theophilou. ${ }^{11}$ His theory was enlarged into the theory of ensembles of excited states by Gross et al ${ }^{12}$.

The eigenvalue problem of the Hamiltonian $\hat{H}$ can be given by

$$
\hat{H} \psi_{k}=E_{k} \psi_{k} \quad(k=1, \ldots, M) .
$$

From the energy eigenvalues,

$$
E_{1} \leq E_{2} \leq \ldots
$$

the ensemble energy,

$$
\mathrm{E}=\sum_{k=1}^{M} w_{k} E_{k},
$$

can be constructed, where $w_{1} \geq w_{2} \geq w_{M} \geq 0$. The generalized Rayleigh-Ritz variational principle is valid $^{12}$ for the ensemble energy. Therefore the generalized Hohenberg-Kohn-theorems can be derived. (i) The external potential $\mathrm{V}(r)$ is determined within a trivial additive constant, by the ensemble density $n$ defined as

$$
n=\sum_{k=1}^{M} w_{k} n_{k} .
$$

(ii) For a trial ensemble density $n^{\prime}(r)$ such that

$$
n^{\prime}(r) \geq 0
$$

and

$$
\int n^{\prime}(\mathbf{r}) \mathrm{d} \mathbf{r}=N,
$$

$$
\mathrm{E}[n] \leq \mathrm{E}\left[n^{\prime}\right],
$$

i.e. the ensemble functional $E$ takes its minimum at the correct ensemble density $n$.

Kohn-Sham equations can also be derived for the ensemble:

$$
\left[-\frac{1}{2} \nabla^{2}+v_{\mathrm{KS}}\left(\mathbf{r} ; w, n_{w}\right)\right] \phi_{i}^{w}(\mathbf{r})=\varepsilon_{i}^{w} \phi_{i}^{w}(\mathbf{r}) .
$$

The ensemble Kohn-Sham potential

$$
\mathrm{V}_{\mathrm{KS}}\left(\mathbf{r} ; w, n_{w}\right)=\mathrm{V}(\mathbf{r})+\mathrm{V}_{c}\left(\mathbf{r} ; w, n_{w}\right)+\mathrm{V}_{x c}\left(\mathbf{r} ; w, n_{w}\right)
$$

is a sum of the external, the ensemble Coulomb and the ensemble exchange-correlation potentials. The ensemble Kohn-Sham equations (15) have exactly the same form as the ground-state equations. The difference is in the fact that because of (11) there are noninteger occupation numbers also and the ensemble exchange-correlation potential depends on the weighting factors as well.

Let us consider an ensemble constructed from the ground and the first excited states. The first excitation energy is given by ${ }^{12}$

$$
\begin{gathered}
E_{2}-E_{1}=\frac{1}{g_{2}} \sum_{j=N+g_{1}}^{N-1+g_{1}+g_{2}} \varepsilon_{j}^{w}-\frac{1}{g_{1}} \sum_{j=N}^{N-1+g_{1}} \varepsilon_{j}^{w}+ \\
\frac{1}{g_{2}}+\left.\frac{\partial E_{x c}[w, n]}{\partial w}\right|_{n=n_{w}},
\end{gathered}
$$

where $g_{1}$ and $g_{2}$ are the degeneracies of the ground and the first excitation states and

$$
0 \leq w \leq 1 / g_{1} \text {. }
$$

$E_{x c}$ is the ensemble exchange-correlation energy. Introducing the notation,

$$
\bar{\varepsilon}_{N}^{w}=\varepsilon_{j}^{w}, \quad \text { if } \quad N \leq j \leq N-1+g_{1}
$$

and

$$
\bar{\varepsilon}_{N+1}^{w}=\varepsilon_{j}^{w}, \quad \text { if } \quad N+g_{1} \leq j \leq N-1+g_{1}+g_{2},
$$

the first excitation energy has the form

$$
E_{2}-E_{1}=\bar{\varepsilon}_{N+1}^{w}-\bar{\varepsilon}_{N}^{w}+\left.\frac{1}{g_{2}} \frac{\partial E_{x c}[w, n]}{\partial w}\right|_{n=n_{w}} .
$$


If both states are non-degenerate $\left(g_{1}=g_{2}=1\right)$,

$$
\bar{\varepsilon}_{N}^{w}=\varepsilon_{N}^{w}
$$

and

$$
\bar{\varepsilon}_{N+1}^{w}+\varepsilon_{N+1}^{w} .
$$

Consequently, the excitation energy takes the form

$$
E_{2}-E_{1}=\varepsilon_{N+1}^{w}-\varepsilon_{N}^{w}+\left.\frac{\partial E_{x c}[w, n]}{\partial w}\right|_{n=n_{w}} .
$$

In the ground state of the $\mathrm{N}$-electron system the lowest $N$ one-energy levels are occupied in the KohnSham scheme. The first excitation corresponds to raising an electron from the highest occupied level to the lowest unoccupied level $N+1$. The first excitation energy is given by the difference of these energy levels plus an extra term arising from the $w$ dependence of the ensemble exchange-correlation energy. All quantities in the right-hand side of (21) and (24) should be calculated with the ensemble density $n_{w}$.

\section{Hardness and excitation energy}

Let us now take the limit $w \rightarrow 0$ in (21). Then the first excitation energy takes the form

$$
E_{2}-E_{1}=\bar{\varepsilon}_{N+1}^{0}-\bar{\varepsilon}_{N}^{0}+\left.\frac{1}{g_{2}} \lim _{w \rightarrow 0} \frac{\partial E_{x c}^{w}}{\partial w}\right|_{n=n_{w}} .
$$

in the degenerate case and

$$
E_{2}-E_{1}=\varepsilon_{N+1}^{0}-\varepsilon_{N}^{0}+\left.\lim _{w \rightarrow 0} \frac{\partial E_{x c}^{w}}{\partial w}\right|_{n=n_{w}} .
$$

in the non-degenerate case. That is, the first excitation energy can be given by the energy difference of the ground-state lowest unoccupied and highest occupied levels plus an extra term coming from the partial derivative of the ensemble exchange-correlation energy with respect to the weighting factor $\mathrm{w}$ in the limit $w \rightarrow 0$.

Comparing (6) and (24) we immediately obtain that

$$
E_{2}-E_{1}=2 \eta_{\mathrm{KS}}+\left.\frac{1}{g_{2}} \lim _{w \rightarrow 0} \frac{\partial E_{x c}^{w}}{\partial w}\right|_{n=n_{w}},
$$

That is, the first excitation energy is the sum of the Kohn-Sham hardness and the partial derivative of the ensemble exchange-correlation energy with respect to the weighting factor $w$ in the limit $w \rightarrow 0$.

Now, we introduce the concept of the ensemble Kohn-Sham hardness,

$$
\eta_{\mathrm{KS}}^{w}=\frac{1}{2}\left(\bar{\varepsilon}_{N+1}^{w}-\bar{\varepsilon}_{N}^{w}\right)
$$

and

$$
\eta_{\mathrm{KS}}^{w}=\frac{1}{2}\left(\varepsilon_{N+1}^{w}-\varepsilon_{N}^{w}\right),
$$

for the degenerate and the nondegenerate cases respectively. Then the first excitation energy is given by

$$
E_{2}-E_{1}=2 \eta_{\mathrm{KS}}^{w}+\left.\frac{1}{g_{2}} \frac{\partial E_{x c}^{w}}{\partial w}\right|_{n=n_{w}} .
$$

Equations (27) and (30), that establish a relationship between the first excitation energy and the KohnSham hardness, are the main results of this paper.

The excitation energy is often approximated by the difference

$$
E_{2}-E_{1} \approx \varepsilon_{N+1}^{0}-\varepsilon_{N}^{0}=2 \eta_{\mathrm{Ks}} .
$$

Generally, it does not lead to an adequate estimation, as the last term in (27) cannot be neglected.

\section{Discussion}

The total energy should be considered as a function of the electron number $N$. There are derivative discontinuities, however. $\partial E=\partial N$ does not exist for integer $N$. Only, one-sided functional derivatives exist:

$$
\mu^{+} \equiv\left(\frac{\partial E}{\partial N}\right)^{+}=\lim _{\varepsilon \rightarrow 0+} \frac{E(N+\varepsilon)-E(N)}{\varepsilon}=-A
$$

and

$$
\mu^{-} \equiv\left(\frac{\partial E}{\partial N}\right)^{-}=\lim _{\varepsilon \rightarrow 0-} \frac{E(N-\varepsilon)-E(N)}{\varepsilon}=-I .
$$

Equations (3), (32) and (33) lead to

$$
\eta=\frac{1}{2} \delta\left(N-N_{0}\right),
$$


Table 1. Experimental first excitation energy and hardness for some neutral atoms (in $\mathrm{eV}$ ).

\begin{tabular}{lcrc}
\hline Atom & Transition & $E_{2}-E_{1}$ & $2 \eta=I-A$ \\
\hline $\mathrm{Be}$ & $2 s \rightarrow 3 s$ & $6 \cdot 62$ & $9 \cdot 00$ \\
$\mathrm{~B}$ & $2 p \rightarrow 3 s$ & $4 \cdot 97$ & $8 \cdot 02$ \\
$\mathrm{C}$ & $2 p \rightarrow 3 s$ & $7 \cdot 48$ & $10 \cdot 00$ \\
$\mathrm{~N}$ & $2 p \rightarrow 3 s$ & $10 \cdot 34$ & $14 \cdot 46$ \\
$\mathrm{O}$ & $2 p \rightarrow 3 s$ & $9 \cdot 50$ & $12 \cdot 16$ \\
$\mathrm{~F}$ & $2 p \rightarrow 3 s$ & $12 \cdot 87$ & $14 \cdot 02$ \\
$\mathrm{Na}$ & $3 s \rightarrow 3 p$ & $2 \cdot 10$ & $4 \cdot 46$ \\
$\mathrm{Mg}$ & $3 s \rightarrow 3 p$ & $3 \cdot 53$ & $7 \cdot 80$ \\
$\mathrm{Al}$ & $3 p \rightarrow 4 s$ & $3 \cdot 14$ & $5 \cdot 54$ \\
$\mathrm{Si}$ & $3 p \rightarrow 4 s$ & $5 \cdot 01$ & $6 \cdot 76$ \\
$\mathrm{Cl}$ & $3 p \rightarrow 4 s$ & $9 \cdot 05$ & $9 \cdot 36$ \\
\hline
\end{tabular}

where $N_{0}$ denotes integer electron number. So as it was emphasized by Cohen, ${ }^{10}$ for systems with discrete spectra the concept of hardness cannot be used for an integer number of electrons. Therefore, instead of the original definition of the hardness, approximations (5) and (6) are applied and proved to be very useful in several applications.

Now we propose another possibility. The first excitation energy is well defined and it can be an appropriate reactivity index instead of the hardness. From (5), (6), (27) and (31) we can suppose that

$$
E_{2}-E_{1} \approx 2 \eta \text {. }
$$

Of course, this approximation cannot be directly checked as the derivative in (3) at integer number of electrons $N$ does not exist. Only the approximations (5) and (6) are available. Then we can also suppose that

$$
E_{2}-E_{1} \approx I-A \text {. }
$$

Contrary to relation (35), approximation (36) can be checked, as experimental values are available. Table 1 presents experimental values of the first excitation energy and the difference $I-A$ for some atoms. ${ }^{2}$ It shows that approximation (36) is very crude. The first excitation energy is systematically smaller than $I-A$. Therefore the extra term in (27) has an important negative contribution.

Turning to the concept of ensemble hardness, we can immediately see from (30) that $\eta_{\mathrm{KS}}^{w}$ and consequently the derivative of the ensemble exchangecorrelation energy with respect to the weighting factor $w$ are different for different values of $w$. However, it can happen that there exists a $w_{0}$ for which

$$
\left.\frac{\partial E_{x c}^{w}}{\partial w}\right|_{n=n_{w 0}}=0 .
$$

Then

$$
E_{2}-E_{1}=2 \eta_{\mathrm{KS}}^{w}
$$

It was found ${ }^{13}$ that using a simple local ensemble potential there exist such a $w_{0}$ for some atoms. The value of $w_{0}$ is 0.0114 for $\mathrm{F}, 0.113$ for $\mathrm{Na}$ and 0.0178 for $\mathrm{Cl}$, respectively, At $w_{0}$ the ensemble hardness gives directly the excitation energy by virtue of (38).

In conclusion, we can state that there is an intimite relationship between the Kohn-Sham hardness and the first excitation energy. The ground-state KohnSham hardness systematically overestimates the first excitation energy. The ensemble Kohn-Sham hardness, on the other hand, can be rather close or even equal to the first excitation energy.

\section{Acknowledgments}

The author wishes to thank Prof. K D Sen for a number of valuable discussions on the general area embraced by the present work. This paper was written in the frame of the Bilateral Intergovermental Scientific and Technological Cooperation between Hungary and India sponsored by the Research an Technological Innovation Foundation and the Ministry of Science and Technology. This work was also supported by the grant OTKA No. T042505.

\section{References}

1. Hohenberg P and Kohn W 1964 Phys. Rev. 136 B864

2. Parr R G and Yang W 1989 Density functional theory of atoms and molecules (New York: Oxford Univ. Press)

3. Dreizler R M and Gross E K U 1990 Density functional theory (Berlin: Springer-Verlag)

4. Further references in Nagy Á 1998 Phys. Rep. 3981

5. Mulliken R S 1934 J. Chem. Phys. 2782

6. Sanderson R T 1951 Science $\mathbf{1 1 4} 670$

7. Parr R G and Pearson R G 1983 J. Am. Chem. Soc. 1057512

8. Yang W and Parr R G 1985 Proc. Natl. Acad. Sci. USA 826723

9. Zahariev FE and Wang Y A 2004 Phys. Rev. A70 042503

10. Cohen M H 1996 in Density functional theory IV; Theory of chemical reactivity (ed. R F Nalewajski) vol. 183, Topics in current chemistry (Berlin: Springer)

11. Theophilou A K 1978 J. Phys. C12 5419

12. Gross E K U, Oliveira L N and Kohn W 1988 Phys. Rev. A37 2805, 2809, 2821

13. Nagy Á 1996 J. Phys. B29 389 